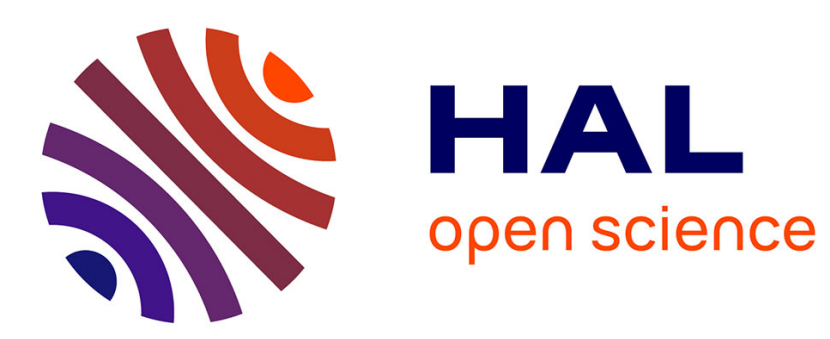

\title{
The Wadge Hierarchy of Petri Nets omega-Languages
}

Jacques Duparc, Olivier Finkel, Jean-Pierre Ressayre

\section{To cite this version:}

Jacques Duparc, Olivier Finkel, Jean-Pierre Ressayre. The Wadge Hierarchy of Petri Nets omegaLanguages. 2013. hal-00743510v2

\section{HAL Id: hal-00743510 https://hal.science/hal-00743510v2}

Preprint submitted on 5 Apr 2013 (v2), last revised 23 Oct 2014 (v3)

HAL is a multi-disciplinary open access archive for the deposit and dissemination of scientific research documents, whether they are published or not. The documents may come from teaching and research institutions in France or abroad, or from public or private research centers.
L'archive ouverte pluridisciplinaire HAL, est destinée au dépôt et à la diffusion de documents scientifiques de niveau recherche, publiés ou non, émanant des établissements d'enseignement et de recherche français ou étrangers, des laboratoires publics ou privés. 


\title{
The Wadge Hierarchy of Petri Nets $\omega$-Languages
}

\section{Jacques Duparc|" and Olivier Finke\| and Jean-Pierre Ressayre ${ }^{\dagger}$}

\begin{abstract}
We describe the Wadge hierarchy of the $\omega$-languages recognized by deterministic Petri nets. This is an extension of the celebrated Wagner hierarchy which turned out to be the Wadge hierarchy of the $\omega$-regular languages. Petri nets are more powerful devices than finite automata. They may be defined as partially blind multi-counter automata. We show that the whole hierarchy has height $\omega^{\omega^{2}}$, and give a description of the restrictions of this hierarchy to partially blind multi-counter automata of some fixed positive number of counters.
\end{abstract}

\section{Introduction}

The languages of infinite words, also called $\omega$-languages, accepted by finite automata were first studied by Büchi to prove the decidability of the monadic second order theory of one successor over the integers. Since then, the $\omega$-regular languages have been thoroughly studied, and many applications to specification and verification of non-terminating systems have been found (see for instance [32, 45, 47] for many results and references). The acceptance of infinite words by other finite machines, such as pushdown automata, multicounter automata, Petri nets, Turing machines, have also been considered, see [4, 10, 22, 45, 46].

The set $\Sigma^{\omega}$ of infinite words over a finite alphabet $\Sigma$ is naturally equipped with the Cantor topology. Then, the complexity of languages of infinite words accepted by finite machines may be studied in terms of topological complexity. This consists in locating these languages at first inside the Borel hierarchy or the projective hierarchy, and then inside the Wadge hierarchy of the Borel subsets. The latter being a huge refinement of the Borel hierarchy. The standard work here can be found in [10, 28, 38, 40, 42, 43, 44, 45, 47].

It is well known that every $\omega$-language accepted by a deterministic Büchi automaton is a $\Pi_{2}^{0}$-set, and that an $\omega$-language accepted by a non-deterministic Büchi (or Muller) automaton is a $\Delta_{3}^{0}$-set. The Borel hierarchy of regular $\omega$-languages is

\footnotetext{
${ }^{*}$ Faculty of Business and Economics, University of Lausanne, CH-1015 - Lausanne

†Équipe de Logique Mathématique, Institut de Mathématiques de Jussieu - Paris Rive Gauche CNRS et Université Paris Diderot, UFR de mathématiques case 7012, 75205 Paris Cedex 13, France
} 
then determined. Moreover Landweber proved that the Borel complexity of any $\omega$-language accepted by a Muller or Büchi automaton can be computed effectively (see [27, 32, 45, 47]).

The trace of the Wadge hierarchy on the $\omega$-regular languages is called the Wagner hierarchy. It has been completely described by Klaus Wagner in [50]. Its length is the ordinal $\omega^{\omega}$. Wagner gave an automaton-like characterization of this hierarchy, based on the notions of chain and superchain, together with an algorithm to compute the Wadge (Wagner) degree of any given $\omega$-regular language. Later, Wilke and Yoo proved that the Wadge degree of an $\omega$-regular language may be computed in polynomial time [51]. This hierarchy was thoroughly studied by Carton and Perrin in [2, 3], and by Victor Selivanov in [34, 35, 39].

There are a large number of classes of finite machines recognizing $\omega$-languages. Each of them yields a countable sub-hierarchy of the Wadge hierarchy. Since the 1980's, and the seminal work of Klaus Wagner on the $\omega$-regular languages - although Wagner was unaware at the time of the connections between the Wadge hierarchy and his own work - the description of these sub-hierarchies has been a constant objective.

The Wadge hierarchy of deterministic context-free $\omega$-languages, together with its length: $\omega^{\left(\omega^{2}\right)}$, was determined in [7, 8]. Whether this hierarchy is decidable or not remains an open problem. The Wadge hierarchy induced by deterministic partially blind 1-counter automata was described in an effective way in [13], and other partial decidability results were obtained in [14]. Then, it was proved in [17, 22] that the Wadge hierarchy of context-free $\omega$-languages and the Wadge hierarchy of effective analytic sets 1 are equal. Intriguingly, the only Wadge class for which the membership of a given context-free $\omega$-language can be decided is the rudimentary $\{\emptyset\}$ (see [14, 15, 16]). In particular, one cannot decide whether a non-deterministic pushdown automaton is universal or not. This latter decision problem is actually $\Pi_{2}^{1}$-complete, hence located at the second level of the projective hierarchy, and therefore it is "highly undecidable" (see [20]). Moreover, the second author showed in [19] that the topological complexity of a context-free $\omega$-language may even depend on the models of set theory. In addition, similar results hold for $\omega$-languages accepted by 2-tape Büchi automata (see for instance [18, 19]). Finally, the Wadge hierarchy of $\omega$-languages of deterministic Turing machines was determined by Victor Selivanov in [36, 37].

Among the many devices that recognize $\omega$-languages and are more powerful than finite automata, there are the Petri nets. They are used to describe distributed systems. Petri nets are directed bipartite graphs, in which the nodes represent

\footnotetext{
${ }^{1}$ The class of effective analytic sets (denoted $\Sigma_{1}^{1}$ ) is the class of all the $\omega$-languages recognized by (non-deterministic) Turing machines.
} 
transitions and places. Any distribution of tokens over the places defines a configuration of the net. Petri nets are related to automata for the reason that they may be regarded as partially blind multicounter automata (see [23]). Petri nets have been extensively examined, particularly in concurrency theory (see for instance [11, 33]). The infinite behavior of Petri nets was first studied by Valk in [48], and of the one of deterministic Petri nets, by Carstensen in [1].

In this paper we first consider some deterministic partially blind multicounter automata (corresponding to deterministic Petri nets) and the $\omega$-languages that they accept once equipped with a Muller acceptance condition. This forms the class of deterministic Petri net $\omega$-languages denoted $\mathcal{L}_{\omega d t}^{3}$ in [1]. We describe the trace of the Wadge hierarchy on these languages, which extends the celebrated Wagner hierarchy of $\omega$-regular languages. We show that the whole hierarchy has height $\omega^{\omega^{2}}$, and we characterize the various restrictions of this hierarchy to some fixed number of partially blind counters.

This article is an extended version of a former conference paper that lacked most of the proofs but which appeared in [9].

\section{Basics on $\omega$-languages, automata, and Petri nets}

We assume the reader to be familiar with the theories of formal languages and $\omega$ regular languages (see for instance [24, 32, 47]). Throughout the paper, we assume $\Sigma$ to be any finite set called the alphabet. A finite word (string) over $\Sigma$ is any sequence of the form $u=a_{1} \ldots a_{k}$, where $k \in \mathbb{N}$, and $a_{i} \in \Sigma$ holds for every $i \leq k$. Notice that when $k=0, u$ becomes the empty word denoted by $\varepsilon$. The length of the word $u$ is denoted by $|u|$ (here $|u|=k$ ). We will write $u(i)=a_{i}$ and $u[i]=u(1) \ldots u(i)$ for $i \leq k$ and $u[0]=\varepsilon$. We will denote by $\Sigma^{*}$ the set of all finite words over $\Sigma$.

An infinite word over $\Sigma$ is some sequence of the form $x=a_{1} a_{2} \ldots a_{n} \ldots$ where each $a_{i}$ (any $i>0$ ) belongs to $\Sigma$. These infinite words are called $\omega$-words for they have length $\omega$ (the first infinite ordinal). An infinite word $x$ over $\Sigma$ can be viewed as a mapping $x: \mathbb{N} \longrightarrow \Sigma$, so we will write $x=x(1) x(2) \ldots$ and $x[n]=x(1) x(2) \ldots x(n)$ for its prefix $2^{2}$ of length $n$. We will write $\Sigma^{\omega}$ for the set of all $\omega$-words over the alphabet $\Sigma$. An $\omega$-language over the alphabet $\Sigma$ is nothing but a subset of $\Sigma^{\omega}$.

As usual, the concatenation of two finite words $u$ and $v$ is denoted by $u v$. The concatenation of a finite word $u$ and an $\omega$-word $x$ gives the $\omega$-words $y=u x$ defined by: $y(k)=u(k)$ if $k \leq|u|$, and $y(k)=x(k-|u|)$ if $k>|u|$. Given any finite

\footnotetext{
${ }^{2}$ note that the enumeration $x=x(1) x(2) \ldots$ does not start at 0 so that we recover the empty word as $x[0]$.
} 
or infinite word $x$, the finite word $u$ is a prefix of $x$ (denoted $u \sqsubseteq x)$ if $u(i)=x(i)$ holds for every nonzero integer $i \leq|u|$.

For any $\omega$-language $V \subseteq \Sigma^{*}$, the $\omega$-power of $V$ is

$$
V^{\omega}=\left\{\sigma=u_{1} \ldots u_{n} \ldots \in \Sigma^{\omega} \mid u_{i} \in V, \forall i \geq 1\right\} .
$$

Definition 1. A finite state machine is a quadruple $\mathcal{M}=\left(Q, \Sigma, \delta, q_{0}\right)$, where $Q$ is a finite set of states, $\Sigma$ is a finite input alphabet, $q_{0} \in Q$ is the initial state, and $\delta$ is a mapping from $Q \times \Sigma$ into $2^{Q}$. It is deterministic if $\delta: Q \times \Sigma \longrightarrow Q$.

Given an infinite word $x$, the infinite sequence of states $\rho=r_{0} r_{1} r_{2} r_{3} \ldots$ is called an (infinite) run of $\mathcal{M}$ on $x$ if both $r_{0}=q_{0}$ and $r_{i+1} \in \delta\left(r_{i}, a_{i}\right)$ holds for all $i \geq 1$. By In $(\rho)$, we denote the set of states that appear infinitely often in $\rho$, namely $\operatorname{In}(\rho)=\left\{q \in Q \mid \forall m \exists n>m r_{n}=q\right\}$.

Equipped with an acceptance condition $F$, a finite state machine becomes a finite state automaton $\mathcal{M}=\left(Q, \Sigma, \delta, q_{0}, F\right)$. It is a Büchi automaton (BA) when $F \subseteq Q$, and $a$ Muller automaton when $F \subseteq 2^{Q}$. A Büchi automaton (respectively a Muller automaton) accepts $x$ if for some infinite run of $\mathcal{M}$ on $x \operatorname{In}(\rho) \cap F \neq \emptyset$ (respectively $\operatorname{In}(\rho) \in F$ holds).

The $\omega$-language accepted by an automaton is the set of all the infinite words that it accepts.

A classical result, essentially due to McNaughton [31], is that given any $\omega$ language $L$, the following are equivalent:

(a) $L$ is accepted by some deterministic Muller automaton,

(b) $L$ is accepted by some non-deterministic Muller automaton,

(c) $L$ is accepted by some non-deterministic Büchi automaton

(d) $L$ is of the form $L=\bigcup_{i \leq n} U_{i} V_{i}^{\omega}$, where each $U_{i}, V_{i}$ are regular languages.

An $\omega$-language that satisfies any of the above conditions is called an $\omega$-regular language (or a regular $\omega$-language). We denote by $R E G_{\omega}$ the class of all $\omega$-regular languages. We now move away from finite automata to consider partially blind multicounter automata.

A multicounter automaton, at each step, may increment or decrement its counters by a fixed amount (a counter is a register that contains an integer). Such a machine is blind if it cannot get any access to the content of its counters during the computation - in other words, if every transition that is allowed when some counter is non-empty, is also available when this counter is empty (and vice versa). On finite words, blind multicounter automata need to empty all their counters in order to accept. This characteristic gives them the power to recognize the language 
of all words that contains the same numbers of 0's and 1's. A multicounter automaton is partially blind if, in addition to being blind, it also gets stuck should one of its counters decrease below zero. Partially blind multicounter automata are strictly more powerful machines than blind multicounter automata. For instance they recognize the language of all the finite words $w$ such that every prefix of $w$ contains no more 0's than 1's (see [23] for more details).

In order to get a partially blind multicounter automaton which accepts the same language as a given Petri net, one can distinguish between the places of a Petri net by dividing them into the bounded ones (the number of tokens in such a place at any time is uniformly bounded) and the unbounded ones. Then each unbounded place may be seen as a partially blind counter, and the tokens in the bounded places determine the state of the partially blind multicounter automaton. The transitions of the Petri net may then be seen as the finite control of the partially blind multicounter automaton and the labels of these transitions are then the input symbols.

On $\omega$-words, allowing $\varepsilon$-transitions does not increase the expressive power of deterministic Petri nets (contrary to what happens with non-deterministic Petri nets, see [1]). For this reason, we restrict ourselves to the sole real time-i.e., $\varepsilon$-transition free - partially blind multicounter automata. Also, there is no loss of generality in assuming that every transition either adds or subtracts 1 or 0 to each counter.

Definition 2. Let $k$ be any nonzero integer. A (real time) deterministic $k$-partially blind-counter machine ( $k$-PBCM) is a 4-tuple

$$
\mathcal{M}=\left(Q, \Sigma, \delta, q_{0}\right)
$$

where $Q$ is a finite set of states, $\Sigma$ is a finite input alphabet, $q_{0} \in Q$ is the initial state, and the transition relation $\delta$ is a partial mapping from $Q \times \Sigma \times\{0,1\}^{k}$ into $Q \times\{0,1,-1\}^{k}$.

A configuration of a $k$-PBCM $\mathcal{M}$ is denoted by $\left(q, c_{1}, \ldots, c_{k}\right)$, where $q \in Q$ is the control state and for every nonzero $i \leq k, c_{i} \in \mathbb{N}$ stands for the content of its ith counter. A $k$-PBCM must satisfy the following conditions:

(a) [Next configuration] Given any $a \in \Sigma$, and $q, q^{\prime} \in Q$ we write $a:\left(q, c_{1}, \ldots, c_{k}\right) \rightarrow \mathcal{M}$ $\left(q^{\prime}, c_{1}+j_{1}, \ldots, c_{k}+j_{k}\right)$ when $\delta\left(q, a, i_{1}, \ldots, i_{k}\right)$ is defined where $i_{m}=1$ if $c_{m}>0$ and $i_{m}=0$ otherwise and $\delta\left(q, a, i_{1}, \ldots, i_{k}\right)=\left(q^{\prime}, j_{1}, \ldots, j_{k}\right)$.

(b) [Blind counter] Whenever $\delta\left(q, a, i_{1}, \ldots, i_{k}\right)$ is defined and $i_{m}=0$ for some $m, 1 \leq m \leq k$ then $\delta\left(q, a, i_{1}, \ldots, i_{m}+1, \ldots, i_{k}\right)$ is defined and $\delta\left(q, a, i_{1}, \ldots, i_{m}, \ldots, i_{k}\right)=\delta\left(q, a, i_{1}, \ldots, i_{m}+1, \ldots, i_{k}\right)$.

(c) [Non-negative counter] If $\delta\left(q, a, i_{1}, \ldots, i_{k}\right)$ is defined and $i_{m}=0$ for some $m, 1 \leq m \leq k$ then $\delta\left(q, a, i_{1}, \ldots, i_{k}\right)=\left(q^{\prime}, j_{1}, \ldots, j_{k}\right)$ with $j_{m} \in\{0,1\}$. 
Following Sheila Greibach in [23] we notice that blind multicounter machines know nothing about their counters (this is what (b) suggests). However, partially blind multicounter machines know something about their counters, namely by substracting 1 and adding 1 they can determine whether they are nonzero. But they cannot determine whether some of their counters are zero. As stated by (c), any transition of a partially blind multicounter machine that would try to decrease one of its counter below zero is undefined, leading the machine to get stuck.

An $\omega$-sequence of configurations $\rho=\left(r_{i}, c_{1}^{i}, \ldots c_{k}^{i}\right)_{i \in \mathbb{N}}$ is called a complete run of $\mathcal{M}$ on an $\omega$-word $x=a_{0} a_{1} a_{2} \ldots a_{n} \ldots$ over $\Sigma$ if

$\circ\left(r_{0}, c_{1}^{0}, \ldots c_{k}^{0}\right)=\left(q_{0}, 0, \ldots, 0\right)$, and for all $i \in \mathbb{N}$

$\circ a_{i}:\left(r_{i}, c_{1}^{i}, \ldots c_{k}^{i}\right) \mapsto \mathcal{M}\left(r_{i+1}, c_{1}^{i+1}, \ldots c_{k}^{i+1}\right)$.

We let $\operatorname{In}(\rho)$ denote the set of all control states visited infinitely often during the complete run $\rho$.

Definition 3. A Büchi (resp. Muller) deterministic k-partially blind-counter automaton is some $k-P B C M \mathcal{M}^{\prime}=\left(Q, \Sigma, \delta, q_{0}\right)$, equipped with an acceptance condition F:

$$
\mathcal{M}=\left(Q, \Sigma, \delta, q_{0}, F\right) .
$$

It is a Büchi (resp. Muller $\left.{ }^{3}\right)$ k-partially blind-counter automaton when $F \subseteq Q$ (resp. $F \subseteq 2^{Q}$ ), and it accepts $x$ if the infinite run of $\mathcal{M}^{\prime}$ on $x$ meets the condition $\operatorname{In}(\rho) \cap F \neq \emptyset$ (respectively $\operatorname{In}(\rho) \in F)$.

We let $L(\mathcal{M})$ denote the $\omega$-language accepted by $\mathcal{M}$. We write $\mathbf{B C}(\mathbf{k})$ for the class of $\omega$-languages accepted by Muller deterministic $k$-partially blind-counter automata.

\section{Borel and Wadge hierarchies}

We assume the reader to be familiar with basic notions of topology and ordinals (in particular the operations of multiplication and exponentiation) that can be found in [26, 28] and in [41].

For any given finite alphabet $X$ - with at least two different letters - we consider the topological space $X^{\omega}$ equipped with the Cantor topology 4 The open sets of $X^{\omega}$ are those of the form $W X^{\omega}$, for some $W \subseteq X^{*}$. The closed sets are the complements of the open sets. The class that contains both the open sets and the

\footnotetext{
${ }^{3}$ The Muller acceptance condition was denoted 3-acceptance in [1 27], and $($ inf,$=)$ in [45].

${ }^{4}$ The product topology of the discrete topology on $X$.
} 
closed sets, and is closed under countable union and intersection is the class of Borel sets. It is nicely set up in a hierarchy by counting how many times these operations are needed.

This defines the Borel Hierarchy: $\boldsymbol{\Sigma}_{1}^{0}$ is the class of open sets , and $\boldsymbol{\Pi}_{1}^{0}$ is the class of closed sets. For any nonzero integer $n, \Sigma_{n+1}^{0}$ is the class of countable unions of sets inside $\boldsymbol{\Pi}_{n}^{0}$, while $\boldsymbol{\Pi}_{n+1}^{0}$ is the class of countable intersections of sets inside $\boldsymbol{\Sigma}_{n}^{0}$. More generally, for any nonzero countable ordinal $\alpha, \boldsymbol{\Sigma}_{\alpha}^{0}$ is the class of countable unions of sets in $\bigcup_{\gamma<\alpha} \Pi_{\gamma}^{0}$, and $\Pi_{\alpha}^{0}$ is the class of countable intersections of sets in $\bigcup_{\gamma<\alpha} \Sigma_{\gamma}^{0}$.

We give without proofs a few basic results about Borel classes. Proofs may be found in [25, 30].

Proposition 1. (a) $\boldsymbol{\Sigma}_{\alpha}^{0} \cup \boldsymbol{\Pi}_{\alpha}^{0} \subsetneq \boldsymbol{\Sigma}_{\alpha+1}^{0} \cap \boldsymbol{\Pi}_{\alpha+1}^{0}$, holds for each countable ordinal $\alpha \geq 1$.

(b) $\bigcup_{\gamma<\alpha} \Sigma_{\gamma}^{0}=\bigcup_{\gamma<\alpha} \Pi_{\gamma}^{0} \subsetneq \Sigma_{\alpha}^{0} \cap \Pi_{\alpha}^{0}$, holds for each countable limit ordinal $\alpha$.

(c) A set $W \subseteq X^{\omega}$ belongs to $\Sigma_{\alpha}^{0}$ iff $X^{\omega} \backslash W$ belongs to $\Pi_{\alpha}^{0}$.

(d) $\boldsymbol{\Sigma}_{\alpha}^{0} \backslash \boldsymbol{\Pi}_{\alpha}^{0} \neq \emptyset$ and $\boldsymbol{\Pi}_{\alpha}^{0} \backslash \boldsymbol{\Sigma}_{\alpha}^{0} \neq \emptyset$ holds for every countable ordinal $\alpha \geq 1$.

The Borel rank of a subset $A$ of $X^{\omega}$ is the least ordinal $\alpha \geq 1$ such that $A$ belongs to $\boldsymbol{\Sigma}_{\alpha}^{0} \cup \boldsymbol{\Pi}_{\alpha}^{0}$.

The Borel hierarchy turns into the very refined Wadge Hierarchy with the use of continuous pre-images.

Definition $4\left(\leq_{w}, \equiv_{w},<_{w}\right)$. We let $X, Y$ be two finite alphabets, and $A \subseteq X^{\omega}, B \subseteq$ $Y^{\omega}, A$ is said Wadge reducible to $B$ (denoted $A \leq_{W} B$ ) if and only if there exists some continuous function $f: X^{\omega} \longrightarrow Y^{\omega}$ that satisfies

$$
\forall x \in X \quad(x \in A \Leftrightarrow f(x) \in B) .
$$

We write $A \equiv_{w} B$ when both $A \leq_{w} B$ and $B \leq_{w} A$ hold, and $A<_{w} B$ when both $A \leq_{w} B$ and $B \not \underline{w}_{w} A$ hold.

We call a set $A \subseteq X^{\omega}$ self dual if $A \equiv_{W} A^{\complement}$ is satisfied (where $A^{\complement}$ stands for the complement of $A$, namely $A^{\complement}=A \backslash X^{\omega}$ ), and non-self dual otherwise 5

\footnotetext{
${ }^{5}$ Non-self dual sets are precisely those that do not satisfy $A \leq_{w} A^{\complement}$.
} 
It is easy to verify that the relation $\leq_{w}$ is both reflexive and transitive, and that $\equiv_{W}$ is an equivalence relation. Now a topological class is a class that is closed under continuous pre-images. Given any set $A$, the class of all its continuous preimages forms a topological class $\Gamma$ called a Wadge class, and $A$ is said to be $\Gamma$ complete since it both belongs to $\Gamma$ and (Wadge) reduces every element in it. It follows that two sets are complete for the same topological class if and only if they are Wadge equivalent. We write $[A]_{W}$ for the Wadge class generated by $A$. So formally

$$
[A]_{W}=\left\{B \subseteq X^{\omega} \mid B \leq_{w} A\right\} .
$$

From Proposition 1] we derive that $\boldsymbol{\Sigma}_{\alpha}^{0}$ (resp. $\boldsymbol{\Pi}_{\alpha}^{0}$ ) is a Wadge class and any set in $\boldsymbol{\Sigma}_{\alpha}^{0} \backslash \boldsymbol{\Pi}_{\alpha}^{0}$ (resp. $\boldsymbol{\Pi}_{\alpha}^{0} \backslash \boldsymbol{\Sigma}_{\alpha}^{0}$ ) is $\boldsymbol{\Sigma}_{\alpha}^{0}$-complete (resp. $\boldsymbol{\Pi}_{\alpha}^{0}$-complete). Both $\boldsymbol{\Sigma}_{n}^{0}$-complete and $\boldsymbol{\Pi}_{n}^{0}$-complete sets (any $0<n<\omega$ ) are examined in [43].

The notion of Wadge reducibility is intricately related to game theory for continuous functions may be regarded as strategies for a given player involved in a two-player game of perfect information and infinite length:

Definition 5. Given any mapping $f: X^{\omega} \longrightarrow Y^{\omega}$, the game $\mathbf{G}(f)$ is a twoplayer game where players take turn picking letters in $X$ for I and $Y$ for II, player I starting the game, and player II being allowed in addition to pass her turn, while player I is not.

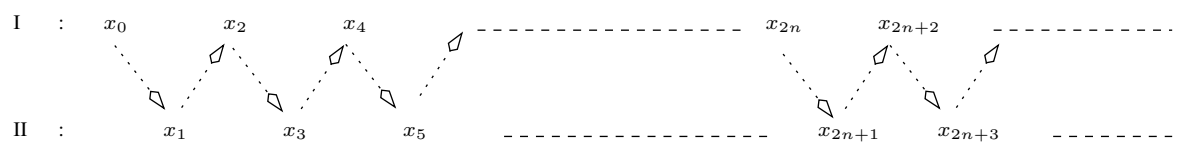

After $\omega$-many moves, player I and player II have respectively constructed $x \in$ $X^{\omega}$ and $y \in Y^{*} \cup Y^{\omega}$. Player II wins the game if $y=f(x)$, otherwise player I wins.

So, in the game $\mathbf{G}(f)$, a strategy for player $I$ is a mapping $\sigma:(Y \cup\{s\})^{\star} \longrightarrow$ $X$, where $s$ is a new letter not in $Y$ that stands for II's moves when she passes her turn ${ }^{6}$. A strategy for player $I I$ is a mapping $f: X^{+} \longrightarrow Y \cup\{s\}$. A strategy is called winning if it ensures a win whatever the opponent does.

This game was designed to characterize the continuous functions.

Theorem 2 (Wadge). Let $f: X^{\omega} \longrightarrow Y^{\omega}$, the following are equivalent:

6" s" stands for "skips". 


\section{$f$ is continuous $\Longleftrightarrow I I$ has a winning strategy in $\mathbf{G}(f)$.}

Proof. This is an easy exercise (see [30, 25]).

Definition 6. For $A \subseteq X^{\omega}$ and $B \subseteq Y^{\omega}$, the Wadge game $\mathbf{W}(A, B)$ is the same game as $\mathbf{G}(f)$, except for the winning condition: II wins if and only if both $y \in Y^{\omega}$ and $(x \in A \Longleftrightarrow y \in B)$ hold.

A winning strategy for $I I$ in $\mathbf{W}(A, B)$ yields a continuous mapping $f: X^{\omega} \longrightarrow$ $Y^{\omega}$ that guaranties $A \leq_{w} B$, whereas any continuous function $f$ that witnesses the reduction relation $A \leq_{w} B$ gives rise to some winning strategy for $I I$ in $\mathbf{G}(f)$ that is also winning for $I I$ in $\mathbf{W}(A, B)$.

Theorem 3 (Wadge). For $A \subseteq X^{\omega}$ and $B \subseteq Y^{\omega}$,

$$
A \leq_{w} B \Longleftrightarrow \text { II has a winning strategy in } \mathbf{W}(A, B) \text {. }
$$

In 1975, Martin proved Borel determinacy [29, 25] which implies that as long as both $A$ and $B$ are both Borel, either player $I$ or player $I I$ has a winning strategy in the Wadge game $\mathbf{W}(A, B)$.

As an immediate consequence we have the following lemma.

Lemma 4 (Wadge). For any finite set $X$, and $A, B \subseteq X^{\omega}$ both Borel,

$$
A \leq_{w} B \text { and } B \not \leq_{w} A \Longrightarrow A \equiv_{w} B^{\complement} \text {. }
$$

Proof. By determinacy, from $A \mathbb{L}_{w} B$, it follows that $I$ has a w.s. in $\mathbf{W}(A, B)$ which gives a w.s. for $I I$ in $\mathbf{W}\left(B^{\complement}, A\right)$ which shows that $B^{\complement} \leq_{w} A$. By the same argument, from $B \leq_{w} A$, one derives $A \leq_{w} B^{\complement}$.

Another immediate consequence of determinacy is Wadge's Lemma.

Lemma 5 (Wadge). For any finite set $X$,

there are no three $\leq_{w}$-incomparable Borel subsets of $X^{\omega}$.

Proof. Assume $A, B, C \subseteq X^{\omega}$ are all Borel $\leq_{w}$-incomparable sets. By Lemma 4 both $A \equiv_{w} B^{\complement}$ and $C \equiv_{w} B^{\complement}$ hold, which leads to $A \equiv_{w} C$.

We recall that a set $S$ is well ordered by the binary relation $<$ on $S$ if and only if $<$ is a linear order on $S$ such that there is no strictly infinite $<$-decreasing sequence of elements from $S$. 
Theorem 6 (Martin-Monk). For $X$ any finite set, there is no sequence $\left(A_{i}\right)_{i \in \omega}$ of Borel subsets of $X^{\omega}$ such that the following holds:

$$
A_{0}>_{w} A_{1}>_{w} A_{2}>_{w} \ldots A_{n}>{ }_{w} A_{n+1}>_{w} \ldots
$$

Proof. See [25, 49].

It follows that up to complementation and $\equiv_{W}$, the class of Borel subsets of $X^{\omega}$, is well-ordered by $<_{w}$. Therefore, there are a unique ordinal $|W H|$ isomorphic to this well-ordering and a mapping $d_{W}^{0}$ from the Borel subsets of $X^{\omega}$ onto $|W H|$, such that for all Borel subsets $A, B$ :

$\circ d_{W}^{0} A<d_{W}^{0} B \Leftrightarrow A<_{w} B$, and

○ $d_{W}^{0} A=d_{W}^{0} B \Leftrightarrow\left(A \equiv_{w} B\right.$ or $\left.A \equiv_{w} B^{\complement}\right)$.

This well-ordering restricted to Borel sets of finite ranks 7 has length the first fixpoint of the operation $\alpha \longrightarrow \omega_{1}^{\alpha}$ [6, 49], where $\omega_{1}$ denotes the first uncountable ordinal.

In order to study the Wadge hierarchy of the class $\mathbf{B C}(\mathbf{k})$ we proceed as in [6] and concentrate on the sole non-self dual sets. For this reason we slightly modify the definition of the Wadge degree.

Definition 7. For $A \subseteq X^{\omega}$, such that $A>{ }_{w} \emptyset$, we set

$\circ d_{w}(\emptyset)=d_{w}\left(\emptyset^{\complement}\right)=1$,

$\circ d_{w}(A)=\sup \left\{d_{w}(B)+1 \mid B\right.$ non-self dual and $\left.B<_{W} A\right\}$.

Every $\omega$-language which is accepted by a deterministic Petri net - more generally by a deterministic $\mathbf{X}$-automaton in the sense of [10] or by a deterministic Turing machine - is a boolean combination of $\boldsymbol{\Sigma}_{2}^{0}$-sets. Therefore its Wadge degree - inside the whole Wadge hierarchy of Borel sets - is less than $\omega_{1}^{\omega}$. Moreover, every nonzero ordinal $\alpha<\omega_{1}^{\omega}$ admits a unique Cantor normal form of base $\omega_{1}$ [41], i.e., it can be written as

$$
\alpha=\omega_{1}^{n_{j}} \cdot \delta_{j}+\omega_{1}^{n_{j-1}} \cdot \delta_{j-1}+\ldots+\omega_{1}^{n_{1}} \cdot \delta_{1}
$$

where $\omega>j>0, \omega>n_{j}>n_{j-1}>\ldots>n_{1} \geq 0$, and $\delta_{j}, \delta_{j-1}, \ldots, \delta_{1}$ are nonzero countable ordinals.

\footnotetext{
${ }^{7}$ The Borel sets of finite ranks are those in $\bigcup_{n \in \mathbb{N}} \boldsymbol{\Sigma}_{n}^{0}=\bigcup_{n \in \mathbb{N}} \boldsymbol{\Pi}_{n}^{0}$.
} 
Since Wagner [50], it is well known that such an ordinal is the Wadge degree of an $\omega$-regular language if and only if the multiplicative coefficients $\left(\delta_{j}, \delta_{j-1}, \ldots, \delta_{1}\right)$ are all integers. It is also known that such an ordinal is the Wadge degree of a deterministic context-free $\omega$-language if and only if these multiplicative coefficients are all below $\omega^{\omega}$ [7]. Our purpose will be to add to the picture the following results:

(a) for every nonzero ordinal $\alpha$ whose Cantor normal form of base $\omega_{1}$ is

$$
\alpha=\omega_{1}^{n_{j}} \cdot \delta_{j}+\omega_{1}^{n_{j-1}} \cdot \delta_{j-1}+\ldots+\omega_{1}^{n_{1}} \cdot \delta_{1}
$$

where the multiplicative coefficients $\delta_{j}, \delta_{j-1}, \ldots, \delta_{1}$ are (nonzero) ordinals less than $\omega^{k+1}$, for some integer $k \geq 1$, there exists some $\omega$-language $L \in$ $\mathbf{B C}(\mathbf{k})$ whose Wadge degree is precisely $\alpha$.

(b) Every non-self dual $\omega$-language in $\mathbf{B C}(\mathbf{k})$ has a Wadge degree of the above form.

In the next section we introduce a few operations over sets of $\omega$-words that will prove extremely useful.

\section{Operations over sets of $\omega$-words}

\subsection{The sum}

Definition 8. For $\left\{X_{+}, X_{-}\right\}$a partition in non-empty sets of $X_{B} \backslash X_{A}$ with $X_{A} \subseteq$ $X_{B}, A \subseteq X_{A}^{\omega}$, and $B \subseteq X_{B}^{\omega}$, we define the addition to be.

$$
B+A=A \cup X_{A}^{*} X_{+} B \cup X_{A}^{*} X_{-} B^{\complement} .
$$

A player in charge of $B+A$ in a Wadge game behaves as a player who begins the play being in charge of $A$, and at any moment may also decide to start anew but being in charge this time of either $B$ or $B^{\complement}$. (The first letter in $X_{B} \backslash X_{A}$ decides between $B$ or $B^{\complement}$ ).

Notice that given any finite alphabets $X, Y$ that contain at least two letters each, and any $B \subseteq X^{\omega}$, there exists $B^{\prime} \subseteq Y^{\omega}$ such that $B \equiv_{w} B^{\prime}$. Moreover, if $B \in \mathbf{B C}(\mathbf{k})$ holds for some fixed integer $k$, then $B^{\prime}$ may be chosen in $\mathbf{B C}(\mathbf{k})$. It follows that by abuse of notation we may write $B+A$ when what we really mean is $B^{\prime}+A$ where $B^{\prime}$ is any set that satisfies both $B^{\prime} \equiv_{w} B$ and $B^{\prime} \subseteq X^{\omega}$ for some alphabet $X$ that strictly extends with at least two new letters the alphabet from which $A$ is taken from. 
Proposition 7 (Wadge). For non-self dual Borel sets $A$ and B,

$$
d_{w}(B+A)=d_{w}(B)+d_{w}(A) .
$$

Notice that this operation is associative: $A+(B+C) \equiv_{W}(A+B)+C$ holds for all non-self dual Borel sets $A, B, C$. Notice also that $(B+A)^{\complement} \equiv_{w} B+A^{\complement}$ is always satisfied. Although $\mathbf{B C}(\mathbf{k})$ is not closed under complementation and $B+A$ was defined with the help of $B^{\complement}$ we will however allow the expression $B+A \in \mathbf{B C}(\mathbf{k})$ for $A, B \in \mathbf{B C}(\mathbf{k})$ as long as there exists some $C \in \mathbf{B C}(\mathbf{k})$ that satisfies $C \equiv_{w} B^{\complement}$.

\subsection{The countable multiplication}

In order to define the countable multiplication of an $\omega$-language, we first define the supremum of a countable family of $\omega$-languages:

Definition 9. For any bijection $f: \mathbb{N} \longrightarrow I$, any family $\left(A_{i}\right)_{i \in I}$ of non-self dual Borel subsets of $X^{\omega}$, and any fixed letter $e \in X$,

$$
\sup _{i \in I} A_{i}=\bigcup_{n \in \mathbb{N}}(X \backslash\{e\})^{n} e A_{f(n)} .
$$

Proposition 8. For $\left(A_{i}\right)_{i \in I}$ any countable family of non-self dual Borel subsets of $X^{\omega}$ with the following property $\forall i \in I \quad \exists j \in I \quad A_{i}<{ }_{w} A_{j}$, we have

(a) $\sup _{i \in I} A_{i}$ is a non-self dual Borel subset of $X^{\omega}$, and

(b) $d_{w}\left(\sup _{i \in I} A_{i}\right)=\sup \left\{d_{w}\left(A_{i}\right) \mid i \in I\right\}$.

Proof. See [6, 7].

By combining the two operations of sum and supremum, we define the multiplication by a countable ordinal.

Definition 10. For $A \subseteq X^{\omega}$, and $0<\alpha<\omega_{1}, A \bullet \alpha$ is inductively defined by:

(a) $A \bullet 1=A$

(b) $A \bullet(\nu+1)=(A \bullet \nu)+A$

(c) $A \bullet \beta=\sup _{\delta \in \beta} A \bullet \delta$ when $\beta$ is a limit ordinal.

By Propositions 7 and 8 , this operation satisfies the following: 
Proposition 9. Let $A \subseteq X^{\omega}$ be some non-self dual Borel set, and $0<\alpha<\omega_{1}$,

$$
d_{w}(A \bullet \alpha)=d_{w}(A) \cdot \alpha .
$$

For a player in charge of $A \bullet \alpha$ in a Wadge game, everything goes as if (s)he could switch again and again between being in charge of $A$ or $A^{\complement}-$ starting anew every time (s)he does so - but restrained from doing so infinitely often by having to construct a decreasing sequence of ordinals $<\alpha$ on the side every time (s)he switches. This operation $A \longrightarrow A \bullet \alpha$ was denoted $A \longrightarrow A^{\wedge} \alpha$ in [8].

\subsection{The multiplication by $\omega_{1}$}

Definition 11. For $A \subseteq X^{\omega}$, and $a, b \notin X$ two different letters, $Y=X \cup\{a, b\}$, $A \bullet \omega_{1} \subseteq(X \cup\{a, b\})^{\omega}$ is defined $\unlhd^{8}$ by

$$
A \bullet \omega_{1}=A \cup Y^{*} a A \cup Y^{*} b A^{\complement} .
$$

Inside a Wadge game, a player in charge of $A \bullet \omega_{1}$ may switch indefinitely between being in charge of $A$ or its complement, deleting what (s)he has already played each time (s)he switches.

Proposition 10. For any non-self dual Borel $A \subseteq X^{\omega}$,

(a) A $\bullet \omega_{1}$ is non-self dual Borel, and

(b) $d_{w}\left(A \bullet \omega_{1}\right)=d_{w}(A) \cdot \omega_{1}$.

Proof. See [6].

The following property will prove very useful.

Proposition 11. If $A \subseteq X^{\omega}$ is regular, then $A \bullet \omega_{1}$ is also regular.

Proof. It follows directly from the definition of $A \bullet \omega_{1}$ and the closure of the class $R E G_{\omega}$ under finite union, complementation, and left concatenation by finitary regular languages [8].

\footnotetext{
${ }^{8}$ This operation was denoted $A^{+}$in [5], $A \longrightarrow A$ : $\infty$ in [8], and $A \longrightarrow A^{\natural}$ in [7].
} 


\subsection{Canonical non-self dual sets}

The empty set is an $\omega$-language with Wadge degree $1\left(d_{w}(\emptyset)=1\right)$. It is non-self dual, its complement has the same Wadge degree $\left(d_{w}(\emptyset)=d_{w}\left(X^{\omega}\right)=1\right)$, and these two are the only $\omega$-languages with degree 1 .

On the basis of the emptyset or its complement, the operations defined above provide non-self dual Borel sets for every Wadge degree $<\omega_{1}^{\omega}$. For convenience, given any $A \subseteq X^{\omega}$ we write $A \bullet \omega_{1}^{n}$ for:

$$
\begin{aligned}
& \circ A \bullet \omega_{1}^{0}=A, \\
& \circ A \bullet \omega_{1}^{n+1}=\left(A \bullet \omega_{1}^{n}\right) \bullet \omega_{1} .
\end{aligned}
$$

By Proposition 10, $d_{w}\left(A \bullet \omega_{1}^{n}\right)=d_{w}(A) \cdot \omega_{1}^{n}$ holds for every non-self dual Borel $A \subseteq X^{\omega}$. It follows immediately that the $\omega$-language $\emptyset \bullet \omega_{1}^{n}$ is a non-self dual Borel set whose Wadge degree is exactly $\omega_{1}^{n}$.

Every nonzero ordinal $\alpha<\omega_{1}^{\omega}$ admits a unique Cantor normal form of base $\omega_{1}$ which is an expression of the form:

$$
\alpha=\omega_{1}^{n_{j}} \cdot \delta_{j}+\omega_{1}^{n_{j-1}} \cdot \delta_{j-1}+\ldots+\omega_{1}^{n_{1}} \cdot \delta_{1} .
$$

where $\omega>j>0, \omega>n_{j}>n_{j-1}>\ldots>n_{1} \geq 0$, and $\delta_{j}, \delta_{j-1}, \ldots, \delta_{1}$ are nonzero countable ordinals [41]. Following [6, 7], by mean of the set theoretical sum and countable multiplication we define:

$$
\Omega(\alpha)=\left(\emptyset \bullet \omega_{1}^{n_{j}}\right) \bullet \delta_{j}+\left(\emptyset \bullet \omega_{1}^{n_{j-1}}\right) \bullet \delta_{j-1}+\ldots+\left(\emptyset \bullet \omega_{1}^{n_{1}}\right) \bullet \delta_{1} .
$$

The result is $d_{w}(\Omega(\alpha))=\alpha$. It follows from Propositions 7, 9, and 10

\section{A hierarchy of $B C(k)$}

From now on, we confine ourselves to the sole ordinals $\alpha<\omega_{1}^{\omega}$ whose Cantor normal forms of base $\omega_{1}$ contain only multiplicative coefficients less than $\omega^{k+1}$. We construct for every such $\alpha$ some Muller deterministic $k$-partially blind-counter automata $\mathcal{M}_{\alpha}$ and $\mathcal{M}_{\alpha}^{-}$such that both $L\left(\mathcal{M}_{\alpha}\right) \equiv_{W} \Omega(\alpha)$ and $L\left(\mathcal{M}_{\alpha}^{-}\right) \equiv_{W} \Omega(\alpha)^{\complement}$ hold.

To start with, notice that for every integer $n$, since $\emptyset \bullet \omega_{1}^{n} \in R E G_{\omega}$ holds, there exists some deterministic Muller automaton $\mathcal{O}_{n}=\left(Q_{n}, X_{n}, \delta_{n}, q_{n}^{0}, \mathcal{F}_{n}\right)$, where $\mathcal{F}_{n} \subseteq 2^{Q_{n}}$ is the collection of designated state sets, such that $L\left(\mathcal{O}_{n}\right)=\emptyset \bullet \omega_{1}^{n}$. 
Proposition 12. For any $\omega$-regular language $A$, any integer $j \geq 1$ there exist $\omega$-languages $B, C \in \mathbf{B C}(\mathbf{j})$ such that

$$
B \equiv_{W}\left(A \bullet \omega^{j}\right) \text { and } C \equiv_{W}\left(A \bullet \omega^{j}\right)^{\complement} .
$$

Proof. Recall that being in charge of $A \bullet \omega^{j}$ in a Wadge game is the same as being able to swap $A$ and $A^{\complement}$, re-starting each time but also constructing step by step at each swapping a strictly decreasing sequence $<\omega^{j}$ on the side. Now any ordinal $\alpha<\omega^{j}$ has a unique Cantor normal form of base $\omega$ :

$$
\alpha=\omega^{j-1} \cdot n_{j-1}+\omega^{j-2} \cdot n_{j-2}+\ldots+\omega^{1} \cdot n_{1}+\omega^{0} \cdot n_{0} .
$$

where $n_{j-1}, n_{j-2}, \ldots, n_{1}, n_{0}$ are (possibly null) integers. Then we see that $j$ many partially blind counters suffice to keep track of the ordinal $\alpha$ : the first counter value is $n_{0}$, the second counter value is $n_{1}, \ldots$, the $j^{\text {th }}$ counter value is $n_{j-1}$.

Let then $A$ be an $\omega$-regular language accepted by a deterministic Muller automaton, and $\mathcal{A}, \mathcal{A}^{-}$be deterministic Muller automata such that $L(\mathcal{A})^{\complement}=L\left(\mathcal{A}^{-}\right)$. For any nonzero integer $j$ we describe deterministic $k$-partially blind counter automata $\mathcal{A}_{\omega^{j}}$ and $\mathcal{A}_{\omega^{j}}^{-}$such that both $L\left(\mathcal{A}_{\omega^{j}}\right) \equiv_{w} L(\mathcal{A}) \bullet \omega^{j}$ and $L\left(\mathcal{A}_{\omega^{j}}^{-}\right) \equiv_{w}$ $\left(L(\mathcal{A}) \bullet \omega^{j}\right)^{\complement}$ hold.

We first add the following new letters to the alphabet of $\mathcal{A}$ (and also $\mathcal{A}^{-}$):

$$
\uparrow_{0}, \uparrow_{1}, \ldots, \uparrow_{j-1}, \downarrow_{0}, \downarrow_{1}, \ldots, \downarrow_{j-1}, a, a^{-} .
$$

We describe $\mathcal{A}_{\omega^{j}}$ (respectively $\mathcal{A}_{\omega^{j}}^{-}$) by the way it behaves when it reads an $\omega$ word. The reader may easily verify that the conditions below may be carried away by a $k$-partially blind counter automaton.

The machine starts reading "up arrow" letters, i.e., those in $\left\{\uparrow_{0}, \uparrow_{1}, \ldots, \uparrow_{j-1}\right\}$ (otherwise it simply rejects). As long as it does so, it increases the content of its counters $-\uparrow_{i}$ stands for an increase by 1 of counter $i$ - but if it does so infinitely often - that is the infinite word $x$ that it reads belongs to $\left\{\uparrow_{0}, \uparrow_{1}, \ldots, \uparrow_{j-1}\right\}^{\omega}-$ the machine $\mathcal{A}_{\omega^{j}}$ rejects while $\mathcal{A}_{\omega^{j}}^{-}$accepts.

Then, after the machine has read the whole prefix of "up arrow" letters, it should encounter either the letter $a_{+}$or the letter $a^{-}$(otherwise it rejects). If it reads $a_{+}$ (resp. $a^{-}$) it starts working just like $\mathcal{A}$ (resp. $\mathcal{A}^{-}$). This goes on until eventually the machine reads a letter inside $\left\{\uparrow_{0}, \uparrow_{1}, \ldots, \uparrow_{j-1}, \downarrow_{0}, \downarrow_{1}, \ldots, \downarrow_{j-1}, a, a^{-}.\right\}$If it is the case, then this letter has to be a "down arrow" of the form $\downarrow_{i}$ followed by "up arrow" letters (possibly none) of the form $\uparrow_{p}$ for some $p<i$, followed by either 
$a_{+}$or $a^{-}$(otherwise both $\mathcal{A}_{\omega^{j}}$ and $\mathcal{A}^{-}{ }_{\omega^{j}}$ rejects). When this word is of the form

$\downarrow_{i}\left(\uparrow_{p}\right)^{l} a$ (resp. $\left.\downarrow_{i}\left(\uparrow_{p}\right)^{l} a^{-}\right)$, it decreases counter $i$ by one and increases counter $p$ by $l$, then it works as $\mathcal{A}$ (resp. $\mathcal{A}^{-}$).

By playing the underlying Wadge games, the reader may easily verify that both $L\left(\mathcal{A}_{\omega^{j}}\right) \equiv_{w} L(\mathcal{A}) \bullet \omega^{j}$ and $L\left(\mathcal{A}_{\omega^{j}}^{-}\right) \equiv_{w}\left(L\left(\mathcal{A}_{\omega^{j}}\right)\right)^{\complement}$ hold.

Proposition 13. For any $\omega$-regular language $A$, any integer $k$, and any ordinal $\omega^{k} \leq \alpha<\omega^{k+1}$, there exist $\omega$-languages $B, C \in \mathbf{B C}(\mathbf{k})$ such that

$$
B \equiv_{W}(A \bullet \alpha) \text { and } C \equiv_{W}(A \bullet \alpha)^{\complement} .
$$

Proof.

(a) If $0<\alpha<\omega$, then from the very definition of the sum, it is immediate to see that if $A$ belongs to $R E G_{\omega}$, then both $A \bullet \alpha$ and its complement belong to $R E G_{\omega}$.

(b) If $\omega \leq \alpha<\omega^{k+1}$, we distinguish between two cases:

(i) If $\alpha=\omega^{k}$, this was examined in Proposition 12

(ii) If $\omega^{k}<\alpha<\omega^{k+1}$, then we consider the Cantor normal form of base $\omega$ of $\alpha$ :

$$
\alpha=\omega^{m_{j}} \cdot n_{j}+\omega^{m_{j-1}} \cdot n_{j-1}+\ldots+\omega^{m_{0}} \cdot n_{0} .
$$

where $k=m_{j}>m_{j-1}>\ldots>m_{0}$ holds, and $n_{j}, n_{j-1}, \ldots, n_{0}$ are all nonzero integers.

By Proposition 12 and case (a), for each $m_{i}(0 \leq i \leq j)$ there are $m_{i^{-}}$ partially blind counter automata $\mathcal{A}_{\omega^{m_{i}}}$ and $\mathcal{A}_{\omega^{m_{i}}}^{-}$such that $L\left(\mathcal{A}_{\omega^{m_{i}}}\right) \equiv_{w}$ $L(\mathcal{A}) \bullet \omega^{m_{i}}$ and $L\left(\mathcal{A}_{\omega^{i}}^{-}\right) \equiv_{w}\left(L\left(\mathcal{A}_{\omega^{i}}\right)\right)^{\complement}$ hold 9 There is no loss of generality in assuming that

(A) for each $i \leq j$, both $\mathcal{A}_{\omega^{m_{i}}}$ and $\mathcal{A}_{\omega^{m_{i}}}^{-}$are $k$-partially blind counter automata whose transition functions only deal with counters of the form $c_{l}$ for $1 \leq l \leq m_{i}$ leaving untouched the counters of the form $c_{m}$ for $m_{i}<m \leq k$.

\footnotetext{
${ }^{9}$ The case $m_{i}=0$ corresponds to 0 -partially blind counter automata $\mathcal{A}_{\omega^{0}}=\mathcal{A}$ and $\mathcal{A}_{\omega^{0}}^{-}$its dual.
} 
(B) The alphabets of the different machines do not overlap. Which means that for $0 \leq h<i \leq j$, if $\Sigma_{h}, \Sigma_{i}$ denote the respective alphabets of $\mathcal{A}_{\omega^{m_{h}}}, \mathcal{A}_{\omega^{m_{i}}}$ (and equivalently of $\mathcal{A}^{-}{ }_{\omega^{m_{h}}}, \mathcal{A}^{-}{ }_{\omega^{m_{i}}}$ ), then $\Sigma_{h} \cap \Sigma_{i}=\emptyset$ holds.

Moreover we set up $\Sigma$ to be $\bigcup_{i \leq j} \Sigma_{i}$, and $\check{\mathcal{A}}_{\omega^{m_{i}}}$ (resp. $\check{\mathcal{A}}_{\omega^{m_{i}}}^{-}$) to be the machine that works on $\Sigma$ - rather than on $\Sigma_{i}$ - just like $\mathcal{A}_{\omega^{m_{i}}}$ (resp. $\left.\mathcal{A}^{-}{ }_{\omega^{m_{i}}}\right)$ as long as it reads letters in $\Sigma_{i}$, and rejects as soon as it reads a letter in $\Sigma \backslash \Sigma_{i}$.

We consider two new letters $t_{+}, t_{-}$for transitions from a machine to another, and we build $\mathcal{A}_{\alpha}, \mathcal{A}_{\alpha}^{-}$as follows.

(A) $\mathcal{A}_{\alpha}$ starts working like $\check{\mathcal{A}}_{\omega^{m_{0}}}$ (resp. $\mathcal{A}_{\alpha}^{-}$starts like $\check{\mathcal{A}}_{\omega^{m_{0}}}^{-}$) as long as no letter $t_{+}$nor $t_{-}$is encountered.

(B) If a letter among $\left\{t_{+}, t_{-}\right\}$is encountered for the $n^{\text {th }}$ time for some $n<n_{0}+\ldots+n_{j}$, we let $i$ be the integer that satisfies

$$
n_{0}+\ldots+n_{i-1}<n+1 \leq n_{0}+\ldots+n_{i}
$$

if $n>n_{0}$, and $i=0$ otherwise. Then right after this letter is read and until another transition letter (either $t_{+}$or $t_{-}$) is eventually read:

○ if this letter is $t_{+}, \mathcal{A}_{\alpha}$ (resp. $\mathcal{A}_{\alpha}^{-}$) works as if it were $\check{\mathcal{A}}_{\omega^{m_{i}}}$, and

$\circ$ if this letter is $t_{-}, \mathcal{A}_{\alpha}$ (resp. $\mathcal{A}_{\alpha}^{-}$) works as if it were $\check{\mathcal{A}}_{\omega^{m_{i}}}^{-}$.

(C) If a letter among $\left\{t_{+}, t_{-}\right\}$is encountered for the $n_{0}+\ldots+n_{j}^{\text {th }}$ time, then $\mathcal{A}_{\alpha}$ (resp. $\mathcal{A}^{-}{ }_{\alpha}$ ) rejects.

The reader should notice that in case (b)((ii))(B) when a machine hits a $t_{+}$or $t_{-}$letter, some of its counters may already have a nonzero content. But this is not a problem since the initial phase of $\check{\mathcal{A}}_{\omega^{m_{i}}}$ (and $\check{\mathcal{A}}_{\omega^{m_{i}}}^{-}$as well) consists in filling up these same counters plus eventually some extra ones. From there, it is tedious but straightforward to verify that both $\mathcal{A}_{\alpha} \equiv_{w} A \bullet \alpha$ and $\mathcal{A}_{\alpha}^{-} \equiv_{w} A \bullet \alpha$ hold.

Theorem 14. Let $\alpha<\omega_{1}^{\omega}$ be any ordinal whose Cantor normal form of base $\omega_{1}$ with multiplicative coefficients less than $\omega^{\omega}$ :

$$
\alpha=\omega_{1}^{n_{j}} \cdot \delta_{j}+\omega_{1}^{n_{j-1}} \cdot \delta_{j-1}+\ldots+\omega_{1}^{n_{0}} \cdot \delta_{0}
$$


where $\omega>j \geq 0, \omega>n_{j}>n_{j-1}>\ldots>n_{0} \geq 0$, and $\omega^{\omega}>\delta_{j}, \delta_{j-1}, \ldots, \delta_{0}>$ 0 .

Let $k$ be the least integer such that $\forall i \leq j \quad \delta_{i}<\omega^{k+1}$. Then there exist $\omega$-languages $B, C \in \mathbf{B C}(\mathbf{k})$ such that

$$
B \equiv_{w} \Omega(\alpha) \text { and } C \equiv_{w} \Omega(\alpha)^{\complement} .
$$

We recall that $\Omega(\alpha)$ is defined by

$$
\Omega(\alpha)=\left(\emptyset \bullet \omega_{1}^{n_{j}}\right) \bullet \delta_{j}+\left(\emptyset \bullet \omega_{1}^{n_{j-1}}\right) \bullet \delta_{j-1}+\ldots+\left(\emptyset \bullet \omega_{1}^{n_{0}}\right) \bullet \delta_{0} .
$$

Proof. For every $i \leq j$ the set $\emptyset \bullet \omega_{1}^{n_{i}}$ is $\omega$-regular, so that for each integer $i \leq j$ we have machines $\mathcal{A}^{i}$ and $\mathcal{A}^{i^{-}}$such that $L\left(\mathcal{A}^{i}\right) \equiv_{w} \emptyset \bullet \omega_{1}^{n_{i}}$ and $L\left(\mathcal{A}^{i^{-}}\right) \equiv_{w}\left(\emptyset \bullet \omega_{1}^{n_{i}}\right)^{\complement}$ hold for every $i \leq j$. The case $j=0$ was already proved in Proposition 13 , so that we may assume that $j>0$ holds.

Now we also consider for each $i \leq j$ the "exact" $k_{i}$-partially blind-counter automata $\mathcal{A}_{\delta_{i}}^{i}$ and $\mathcal{A}_{\delta_{i}}^{i^{-}}$that were designed in the proof of Proposition 13 . (Notice that $k_{i}$ was defined as the least integer such that $\delta_{i}<\omega^{k_{i}+1}$.) We then form for each $i \leq j$, some $k$-partially blind-counter automata $\hat{\mathcal{A}}_{\delta_{i}}^{i}$ and $\hat{\mathcal{A}}_{\delta_{i}}^{i^{-}}$which work exactly the way $\mathcal{A}_{\delta_{i}}^{i}$ and $\mathcal{A}_{\delta_{i}}^{i^{-}}$do on the first $k_{i}$-many counters, leaving untouched the last $k-k_{i}$-many ones .

For simplicity - and without loss of generality - we may assume that both $\Sigma_{h} \cap \Sigma_{i}=\emptyset$ and $\Sigma_{i}=\Sigma_{i}^{-}$hold for every $0 \leq h<i \leq j$, where $\Sigma_{i}, \Sigma_{i}^{-}$stand for the respective alphabets of $\hat{\mathcal{A}}_{\delta_{i}}^{i}$ and $\hat{\mathcal{A}}_{\delta_{i}}^{i^{-}}$.

Then, for each $i \leq j$ we form, some $k$-partially blind-counter automata $\check{\mathcal{A}}_{\delta_{i}}^{i}$ and $\check{\mathcal{A}}_{\delta_{i}}^{i^{-}}$which work on the alphabet $\Sigma=\bigcup_{i \leq j} \Sigma_{i}$. The machine $\check{\mathcal{A}}_{\delta_{i}}^{i}$ (resp. $\check{\mathcal{A}}_{\delta_{i}}^{i^{-}}$) works as $\hat{\mathcal{A}}_{\delta_{i}}^{i}$ (resp. $\hat{\mathcal{A}}_{\delta_{i}}^{i^{-}}$) on the alphabet $\Sigma_{i}$, and both machines reject if they eventually read a letter not in $\Sigma_{i}$.

At last we use new letters $t_{+}, t_{-}$for getting from one machine to another, and we build $\mathcal{A}_{\alpha}, \mathcal{A}_{\alpha}^{-}$as follows.

(a) As long as neither $t_{+}$nor $t_{-}$is encountered, $\mathcal{A}_{\alpha}$ (resp. $\mathcal{A}_{\alpha}^{-}$) works as $\check{\mathcal{A}}_{\delta_{0}}^{0}$ (resp. $\check{\mathcal{A}}_{\delta_{0}}^{0^{-}}$).

(b) If a letter among $\left\{t_{+}, t_{-}\right\}$is encountered for the $i^{\text {th }}$ time for some $i<j$, then right after this letter is read and until another letter of the form $t_{+}$or $t_{-}$ is eventually read:

$\circ$ if this letter is $t_{+}, \mathcal{A}_{\alpha}$ (resp. $\mathcal{A}_{\alpha}^{-}$) now behaves as if it were $\check{\mathcal{A}}_{\delta_{i}}^{i}$, and 
$\circ$ if this letter is $t_{-}, \mathcal{A}_{\alpha}$ (resp. $\mathcal{A}_{\alpha}^{-}$) now behaves as if it were $\check{\mathcal{A}}_{\delta_{i}}^{i^{-}}$.

(c) If a letter among $\left\{t_{+}, t_{-}\right\}$is encountered for the $j^{\text {th }}$, then both $\mathcal{A}_{\alpha}$ and $\mathcal{A}_{\alpha}^{-}$ reject right away.

The result is $L\left(\mathcal{A}_{\alpha}\right) \equiv_{w} \Omega(\alpha)$ and $L\left(\mathcal{A}_{\alpha}^{-}\right) \equiv_{w} \Omega(\alpha)^{\complement}$. We leave the tedious but straightforward details to the reader.

\section{Localisation of $B C(k)$}

This section is dedicated to proving that no other Wadge class than the ones described in Theorem 14 is generated by some non-self dual $\omega$-language in $B C(k)$. As a preliminary, we present a technical result about the Wadge hierarchy, a few others about ordinal combinatorics, and some notations.

For any $A \subseteq X^{\omega}$ and $u \in X^{*}$, we write $u^{-1} A$ for the set $\left\{x \in X^{\omega} \mid u x \in A\right\}$, and we say that $A$ is initializable if the second player has a w.s. in the Wadge game $\mathbf{W}(A, A)$ even though she is restricted to positions $u \in X^{*}$ that satisfy $u^{-1} A \equiv_{w} A$.

Lemma 15. For $A \subseteq X^{\omega}$ any initializable set, $B \subseteq Y^{\omega}$, and $\delta, \theta$ any countable ordinals,

$$
A \bullet(\theta+1) \leq_{w} B \leq_{w} A \bullet \delta \Longrightarrow \exists u \in Y^{*}\left\{\begin{array}{c}
u^{-1} B \equiv_{w} A \bullet(\theta+1) \\
\text { or } \\
u^{-1} B \equiv_{w}(A \bullet(\theta+1))^{\complement} .
\end{array}\right.
$$

Proof. The case $\theta+1=\delta$ is obvious since the empty word works for $u$. So in the sequel we assume $\theta+1<\delta$. The proof goes by induction on $\delta$.

\section{Assume $\delta$ is limit.}

(a) If $B \equiv_{w} A \bullet \delta$, then clearly the set

$$
\left\{d_{W}^{0} u^{-1} B: u \in Y^{*} \text { and } u^{-1} B<_{w} B\right\}
$$

is unbounded in $d_{W}^{0}(A \bullet \delta)=d_{W}^{0}(A) \cdot \delta$. Hence there exists some ordinal $\xi<\delta$ and some $v \in Y^{*}$ that both satisfy

$$
A \bullet(\theta+1) \leq_{w} v^{-1} B \leq_{w}(A \bullet \xi)<_{w} A \bullet \delta
$$


Then by induction hypothesis one gets some $u^{\prime} \in Y^{*}$ such that

$$
\left\{\begin{array}{c}
u^{\prime-1} v^{-1} B \equiv_{w} A \bullet(\theta+1) \\
u^{\prime-1} v^{-1} B \equiv_{w}(A \bullet(\theta+1))^{\complement}
\end{array}\right.
$$

Hence $u=v u^{\prime}$ works.

(b) If $B<{ }_{w} A \bullet \delta$, then $d_{W}^{0}(B)<d_{W}^{0}(A \bullet \delta)=d_{W}^{0}(A) \cdot \delta$. Hence, for some $\xi<\delta$ we have

$$
A \bullet(\theta+1) \leq_{w} B \leq_{w}(A \bullet \xi)
$$

which gives the result using the induction hypothesis on $\xi$.

\section{Assume $\delta$ is successor.}

(a) Assume $\delta=\zeta+2$.

(i) Assume $(A \bullet(\zeta+1))^{\complement} \leq_{w} B \leq_{w} A \bullet(\zeta+2)$.

We consider the following combination of Wadge games with 3 players : $I, I I$ and $I I I$ :

$\circ I$ is in charge of $\left((A \bullet \zeta)+A^{\complement}\right)-$ which is Wadge equivalent to $(A \bullet(\zeta+1))^{\complement}$,

○ II is in charge of $B$, and

○ III is in charge of $A \bullet(\zeta+2)$.

$I I$ applies a w.s. that reduces $I$ and $I I I$ applies a w.s. that reduces $I I$. This means that if $I$ plays $x_{1}, I I$ plays $x_{2}$ and III plays $x_{3}$ then II reduces $I$ if $x_{1} \in(A \bullet \zeta)+A^{\complement} \Longleftrightarrow x_{2} \in B$; and III reduces $I I$ if $x_{3} \in A \bullet(\zeta+2) \Longleftrightarrow x_{2} \in B$.

Assume now that player $I$ remaining in the right tail $A^{\complement}$ (i.e. without going into $(A \bullet \zeta)$ or $\left.(A \bullet \zeta)^{\complement}\right)$ applies a winning strategy in the Wadge game $W\left(A^{\complement}, A\right)$ against Player III as long as III stays in the tail part $A$ of $A \bullet(\zeta+1)+A$. Necessarily after a finite number of moves player $I I I$ exits the right most $A$ and chooses $(A \bullet(\zeta+1))^{\complement}$ - for the other choice $A \bullet(\zeta+1)$ would be a losing one. We let $v$ be the position of player $I I$ at that point, so that we obtain:

$$
(A \bullet(\zeta+1))^{\complement} \leq_{w} v^{-1} B \leq_{w}(A \bullet(\zeta+1))^{\complement},
$$

hence

$$
v^{-1} B \equiv_{w}(A \bullet(\zeta+1))^{\complement}
$$


If $\theta+1=\zeta+1$ we are done. Otherwise we have

$$
(A \bullet(\theta+1))^{\complement} \leq_{w} v^{-1} B^{\complement} \leq_{w} A \bullet(\zeta+1)
$$

By induction hypothesis there exists $u$ extending $v$ such that

$$
u^{-1} B^{\complement} \equiv_{w} A \bullet(\theta+1) \text { or } u^{-1} B^{\complement} \equiv_{w}(A \bullet(\theta+1))^{\complement}
$$

which gives

$$
u^{-1} B \equiv_{w} A \bullet(\theta+1) \text { or } u^{-1} B \equiv_{w}(A \bullet(\theta+1))^{\complement} .
$$

(ii) Assume $B \leq_{w} A \bullet(\zeta+1)$.

Since $\zeta+1<\delta$ holds, the result relies on the induction hypothesis for

$$
A \bullet(\theta+1) \leq_{w} B \leq_{w} A \bullet(\zeta+1) .
$$

(b) Assume $\delta=\zeta+1, \zeta$ limit:

If $B \leq_{w} A \bullet \zeta$ holds the result follows from the induction hypothesis. Thus we assume that the following holds:

$$
(A \bullet \zeta)^{\complement} \leq_{w} B \leq_{w}(A \bullet \zeta)+A
$$

Now consider the following combination of Wadge games with 3 players where:

$\circ I$ is in charge of $(A \bullet \theta)+A+A+A^{\complement}$ (this is Wadge equivalent to $\left.(A \bullet(\theta+3))^{\complement}\right)$,

$\circ I I$ is in charge of $B$, and

○ III is in charge of $A \bullet \zeta+A$.

II applies a w.s. that reduces $I$ and III applies a w.s. that reduces II. Player $I$ applies a winning strategy in the Wadge game $W\left(A^{\complement}, A\right)$ against Player III as long as Player III remains in the tail part $A$ of $(A \bullet \zeta)+A$. Necessarily after a finite number of moves player III exits the first $A$ and chooses $(A \bullet \zeta)^{\complement}$ or $(A \bullet \zeta)$.

Now notice that since $A$ is non-self dual, the set $\left\{w \in X^{*} \mid w^{-1} A \equiv_{w}\right.$ $A\}$ is a tree - it is closed under prefixes - that contains an infinite branch. We let $x$ be such an infinite branch.

○ If $x \in A$, then player $I$ chooses to go into $(A \bullet \theta+A+A)^{\complement}$ if III chooses $(A \bullet \zeta)^{\complement}$ and into $(A \bullet \theta+A+A)$ if III chooses $(A \bullet \zeta)$.

○ If $x \notin A$, then player $I$ chooses to go into $(A \bullet \theta+A+A)$ if III chooses $(A \bullet \zeta)^{\complement}$ and into $(A \bullet \theta+A+A)^{\complement}$ if III chooses $(A \bullet \zeta)$. 
Then $I$ plays along $x$, so that $I I I$ is forced to choose $A \bullet \gamma$ for some $\gamma<\zeta$ (by definition $A \bullet \zeta=\sup _{\gamma<\zeta} A \bullet \gamma$ ). After III makes that choice, $I I$ is in a position $v$ that satisfies

$A \bullet(\theta+2)^{\complement} \leq_{w} v^{-1} B \leq_{w} A \bullet \gamma$ or $A \bullet(\theta+2) \leq_{w} v^{-1} B \leq_{w} A \bullet \gamma$.

Therefore we obtain

$$
A \bullet(\theta+1) \leq_{w} v^{-1} B \leq_{w} A \bullet \gamma
$$

which gives the result by induction hypothesis since $\gamma<\delta$ holds.

Lemma 16. We let $B \subseteq Y^{\omega}, A \subseteq X^{\omega}$ be any initializable set, and $\delta, \theta$ be any countable ordinals. We consider any set of the form

$$
C=A \bullet \omega_{1}^{n} \bullet \nu_{n}+\ldots+A \bullet \omega_{1}^{n-1} \bullet \nu_{n-1}+\ldots+A \bullet \omega_{1} \bullet \nu_{1}
$$

for any nonzero integer $n$, and countable coefficients $\nu_{n}, \nu_{n-1}, \ldots, \nu_{1}$ with at least one of them being nonzero.

$C+A \bullet(\theta+1) \leq{ }_{w} B \leq{ }_{w} C+A \bullet \delta \Longrightarrow \exists u \in Y^{*}\left\{\begin{array}{c}u^{-1} B \equiv_{w} C+A \bullet(\theta+1) \\ \text { or } \\ u^{-1} B \equiv_{w}(C+A \bullet(\theta+1))^{\complement} .\end{array}\right.$

Proof. The proof is very similar to the one of Lemma 15, so we leave it to the reader.

We recall that for any set of ordinals $\mathcal{O}$, its order type - denoted $\operatorname{ot}(\mathcal{O})$ - is the unique ordinal that is isomorphic to $\mathcal{O}$ ordered by membership (which is nothing but the usual ordering on ordinals).

Definition 12. The function $\mathcal{H}: \omega^{\omega} \times \omega^{\omega} \longrightarrow$ On is defined by

$$
\mathcal{H}(\alpha, \beta)=\omega^{k} \cdot\left(l_{k}+m_{k}\right)+\omega^{k-1} \cdot\left(l_{k-1}+m_{k-1}\right)+\ldots+\omega^{0} \cdot\left(l_{0}+m_{0}\right) .
$$

Where (a variation of the) the Cantor normal form of base $\omega$ of $\alpha$ (resp. $\beta$ ) is

$$
\begin{gathered}
\alpha=\omega^{k} \cdot l_{k}+\omega^{k-1} \cdot l_{k-1}+\ldots+\omega^{0} \cdot l_{0} \\
\beta=\omega^{k} \cdot m_{k}+\omega^{k-1} \cdot m_{k-1}+\ldots+\omega^{0} \cdot m_{0}
\end{gathered}
$$

with $l_{k}, m_{k}, l_{k-1}, m_{k-1}, \ldots, l_{0}, m_{0} \in \mathbb{N}$. (Some of these integers may be null ${ }^{10}$ )

\footnotetext{
${ }^{10}$ In particular, $l_{k}, l_{k-1}, \ldots m_{k}, m_{k-1}, \ldots$ might be null, but since $\alpha, \beta>0$ holds, at least one of the $l_{i}$ 's, and one of the $m_{i}$ 's are different from zero.
} 
Lemma 17. Let $\mathcal{H}: \omega^{\omega} \times \omega^{\omega} \longrightarrow O n, 0<\alpha^{\prime}, \alpha, \beta^{\prime} \beta<\omega^{\omega}$ with $\alpha^{\prime} \leq \alpha, \beta^{\prime} \leq \beta$ but either $\alpha^{\prime}<\alpha$ or $\beta^{\prime}<\beta$, then

$$
\mathcal{H}\left(\alpha^{\prime}, \beta^{\prime}\right)<\mathcal{H}(\alpha, \beta) .
$$

Proof. This is tedious but straightforward, and left to the reader.

We make use of the mapping $\mathcal{H}$ to prove the following combinatorial result.

Lemma 18. Let $\alpha, \beta, \gamma$ be nonzero ordinals with $\alpha, \beta<\omega^{\omega}$, and $f: \gamma \longrightarrow\{0,1\}$.

If both $\alpha=\operatorname{ot}\left(f^{-1}[0]\right)$ and $\beta=\operatorname{ot}\left(f^{-1}[1]\right)$ hold, then $\gamma \leq \mathcal{H}(\alpha, \beta)$.

Proof. The proof goes by induction on $(\max \{\alpha, \beta\}, \min \{\alpha, \beta\})$ ordered by lexicographic ordering.

(a) If $\alpha=\beta=1$, then the result is immediate.

(b) We assume $\alpha \geq \beta$, and we let $(\alpha, \beta)$ be the $<_{\text {lex }}$-least pair such that there exists some ordinal $\gamma$ together with $f: \gamma \longrightarrow\{0,1\}$ that satisfy

○ $\alpha=o t\left(f^{-1}[0]\right)$,

$\circ \beta=o t\left(f^{-1}[1]\right)$, and

$\circ \gamma>\mathcal{H}(\alpha, \beta)$.

We consider the order types of the following two sets of ordinals:

(i) $\alpha^{\prime}=o t(\{\theta<\mathcal{H}(\alpha, \beta) \mid f(\theta)=0\})$, and

(ii) $\beta^{\prime}=o t(\{\theta<\mathcal{H}(\alpha, \beta) \mid f(\theta)=1\})$,

together with $f^{\prime}$ the restriction of $f$ to $\mathcal{H}(\alpha, \beta)$. Necessarily either $\alpha^{\prime}<\alpha$ or $\beta^{\prime}<\beta$ holds. Therefore we have

$$
\left(\max \left\{\alpha^{\prime}, \beta^{\prime}\right\}, \min \left\{\alpha^{\prime}, \beta^{\prime}\right\}\right)<_{\text {lex }}(\max \{\alpha, \beta\}, \min \{\alpha, \beta\}) .
$$

Hence we get the ordinal $\gamma^{\prime}=\mathcal{H}(\alpha, \beta)$, together with the mapping $f^{\prime}$ : $\mathcal{H}(\alpha, \beta) \longrightarrow\{0,1\}$ such that $\alpha^{\prime}=\operatorname{ot}\left(\left\{\theta<\gamma^{\prime} \mid f^{\prime}(\theta)=0\right\}\right)$ and $\beta^{\prime}=$ $\operatorname{ot}\left(\left\{\theta<\gamma^{\prime} \mid f^{\prime}(\theta)=1\right\}\right)$. By Lemma 17, we obtain $\gamma^{\prime}=\mathcal{H}(\alpha, \beta)>$ $\mathcal{H}\left(\alpha^{\prime}, \beta^{\prime}\right)$ which contradicts the induction hypothesis. 
Corollary 19. Let $k, n$ be nonzero integers, $\gamma$ be any ordinal, $0 \leq \alpha_{0}, \ldots, \alpha_{k}<$ $\omega^{n}$, and $f: \gamma \longrightarrow\{0, \ldots, k\}$.

$$
\text { If } \forall i \leq k \quad \alpha_{i}=\operatorname{ot}\left(f^{-1}[i]\right) \text { holds, then } \gamma<\omega^{\mathrm{n}} .
$$

Proof. This is immediate from Lemma 18 .

Lemma 20. Let $k$ be some nonzero integer, $\left(\mathbb{N}^{k}, \lesssim\right)$ be a well-ordering such that

$$
\left(a_{0}, \ldots, a_{k-1}\right) \lesssim\left(b_{0}, \ldots, b_{k-1}\right) \Longrightarrow\left\{\begin{array}{c}
\forall i<k \quad a_{i} \leq b_{i} \\
\text { or } \\
\exists i, j<k \quad a_{i}<b_{i} \text { and } a_{j}>b_{j}
\end{array}\right.
$$

holds for every $k$-tuples $\left(a_{0}, \ldots, a_{k-1}\right),\left(b_{0}, \ldots, b_{k-1}\right) \in \mathbb{N}^{k}$. Then, the order type of $\left(\mathbb{N}^{k}, \lesssim\right)$ is at most $\omega^{k}$.

Proof. The proof goes by induction on $k \geq 1$.

(a) The initial case $k=1$ is immediate since $\left(\mathbb{N}^{1}, \lesssim\right)$ is nothing but the usual ordering on integers.

(b) We assume the result holds for $k \geq 1$, and we show that it holds for $k+1$.

Claim 21. For any integer $n$, the order type of the following set (ordered by $\lesssim$

$$
A_{n}=\left\{\left(a_{0}, a_{1}, \ldots, a_{k}\right) \in \mathbb{N}^{k+1} \mid\left(a_{0}, a_{1}, \ldots, a_{k}\right)<(n, n, \ldots, n)\right\}
$$

is strictly below $\omega^{(k+1)}$.

Proof. Notice that if $\left(a_{0}, a_{1}, \ldots, a_{k}\right)<(n, n, \ldots, n)$ holds then $a_{i}<n$ must hold for some $i \leq k+1$.

For each $i \leq k+1$ and each $j<n$ we consider

$$
A_{(i, j)}=\left\{\left(a_{0}, a_{1}, \ldots, a_{k}\right) \in \mathbb{N}^{k+1} \mid a_{i}=j\right\},
$$

and $\alpha_{(i, j)}=\operatorname{ot}\left(A_{(i, j)}\right)$ its order type (ordered by $\lesssim$ ). Notice that for

$$
\left(a_{0}, \ldots, a_{i-1}, j, a_{i+1}, \ldots, a_{k}\right) \lesssim\left(b_{0}, \ldots, b_{i-1}, j, b_{i+1}, \ldots, b_{k}\right)
$$




$$
\left\{\begin{array}{c}
\forall l \in\{0, \ldots, i-1, i+1, \ldots, k\} \quad a_{l} \leq b_{l} \\
\text { or } \\
\exists l, m \in\{0, \ldots, i-1, i+1, \ldots, k\} \quad a_{l}<b_{l} \text { and } a_{m}>b_{m}
\end{array}\right.
$$

Therefore by induction hypothesis, $\alpha_{(i, j)}<\omega^{k+1}$ holds for all $i \leq k+1$ and $j<n$. It follows from Corollary 19 that $o t\left(A_{n}\right)<\omega^{k+1}$ holds.

On the other hand for every integer $n \geq 0$ it holds that $(n, n, \ldots, n)<$ $(n+1, n+1, \ldots, n+1)$. Moreover if $n=\max \left\{a_{0}, a_{1}, \ldots, a_{k}\right\}+1$ then $\left(a_{0}, a_{1}, \ldots, a_{k}\right)<(n, n, \ldots, n)$. Therefore the sequence of ordinals $\left(\text { ot }\left(A_{n}\right)\right)_{n \geq 1}$ is cofinal in $o t\left(\mathbb{N}^{k+1}\right)$ and thus the order type of $\left(\mathbb{N}^{k+1}, \lesssim\right)$ is at most $\omega^{k+1}$.

Lemma 22. We let $k$ be any nonzero integer, $B \in \mathbf{B C}(\mathbf{k}), A \subseteq X^{\omega}$ be any initializable set, and $\delta$ any countable ordinal.

$$
B \leq_{w} A \bullet \delta \Longrightarrow B \leq_{w} A \bullet \alpha \text { for some } \alpha<\omega^{k+1} .
$$

Notice that an immediate consequence is that $B \equiv_{w} A \bullet \delta$ holds only for ordinals $\delta<\omega^{k+1}$.

Proof. First notice that for every $B \subseteq X^{\omega}$, and every $u \in X^{*}$, if $B \in \mathbf{B C}(\mathbf{k})$ holds, then $u^{-1} B \in \mathbf{B C}(\mathbf{k})$ holds too.

Towards a contradiction, we assume that $A \bullet \alpha<_{w} B \leq_{w} A \bullet \delta$ holds for all $\alpha<\omega^{k+1}$. We let $\mathcal{B}$ be a $k$-partially blind counter automaton that recognizes $B$. By Lemma 15. for each successor ordinal $\alpha<\omega^{k+1}$ there exists some $u_{\alpha} \in X^{*}$ such that $u_{\alpha}^{-1} B \equiv_{w} A \bullet \alpha$ or $u_{\alpha}^{-1} B \equiv_{w}(A \bullet \alpha)^{\complement}$. For each such $u_{\alpha}$, we form $\left(q_{\alpha}, c_{\alpha, 0}, c_{\alpha, 1}, \ldots, c_{\alpha, k-1}\right)$ where $q_{\alpha}$ denotes the control state that $\mathcal{B}$ is in after having read $u_{\alpha}$, and $c_{\alpha, i}$ the height of its counter number $i$ (any $i<k$ ).

Now there exists necessarily some control state $q$ such that

$$
\text { the order type of the set } S=\left\{\alpha<\omega^{k+1} \mid \alpha \text { successor and } q_{\alpha}=q\right\} \text { is } \omega^{k+1} \text {. }
$$

Now, by Lemma 20 there exist $\alpha, \alpha^{\prime} \in S$ such that $\alpha^{\prime}<\alpha$ holds together with $c_{\alpha, i} \leq c_{\alpha, i}^{\prime}$ holds for all $i<k$. (Without loss of generality, we may even assume that $\omega \leq \alpha^{\prime}<\alpha$ holds.) Let us denote $\mathcal{B}_{\alpha}^{\prime}$ the $k$-partially blind counter automaton $\mathcal{B}$ that starts in state $\left(q_{\alpha^{\prime}}, c_{\alpha^{\prime}, 0}, c_{\alpha^{\prime}, 1}, \ldots, c_{\alpha^{\prime}, k-1}\right)$, and $\mathcal{B}_{\alpha}$ the one that starts in state $\left(q_{\alpha}, c_{\alpha, 0}, c_{\alpha, 1}, \ldots, c_{\alpha, k-1}\right)$. Notice that since $c_{\alpha, i} \leq c_{\alpha, i}^{\prime}$ holds for all $i<k$, 
$\mathcal{B}_{\alpha^{\prime}}$ performs exactly the same as $\mathcal{B}_{\alpha}$ except when the latter crashes for it tries to decrease a counter that is already empty. But it is then not difficult to see that given the above assumption - that $\omega \leq \alpha^{\prime}<\alpha$ holds $-u_{\alpha}^{-1} B \leq{ }_{w} u_{\alpha^{\prime}}^{-1} B$ holds which leads to either $A \bullet \alpha \leq_{w} A \bullet \alpha^{\prime}$ or $(A \bullet \alpha)^{\complement} \leq_{w} A \bullet \alpha^{\prime}$. In both cases, it contradicts $\alpha^{\prime}<\alpha$.

Notice that the set $\emptyset \bullet \omega_{1}^{n}$ is initializable, so we have in particular the following result.

Lemma 23. For $k, n$ any integers, $A$ any non-self dual $\omega$-language in $\mathbf{B C}(\mathbf{k})$, and any nonzero countable ordinal $\alpha$,

$$
A \text { or } A^{\complement} \equiv_{w}\left(\emptyset \bullet \omega_{1}^{n}\right) \bullet \alpha \Longrightarrow \alpha<\omega^{k+1} .
$$

In a similar way, we can now state the following lemma.

Lemma 24. We let $k$ be any nonzero integer, $B \in \mathrm{BC}(\mathbf{k}), A \subseteq X^{\omega}$ be any initializable set, $\delta$ be any countable ordinal, and $C$ be any set of the form

$$
C=A \bullet \omega_{1}^{n} \bullet \nu_{n}+\ldots+A \bullet \omega_{1}^{n-1} \bullet \nu_{n-1}+\ldots+A \bullet \omega_{1} \bullet \nu_{1}
$$

for any nonzero integer $n$, and countable multiplicative coefficients $\nu_{n}, \nu_{n-1}, \ldots, \nu_{1}$ with at least one of them being nonzero.

$$
B \leq_{w} C+A \bullet \delta \Longrightarrow B \leq_{w} C+A \bullet \alpha \text { for some } \alpha<\omega^{k+1} .
$$

Proof. The proof is very similar to the one of Lemma 22, so we leave it to the reader.

Theorem 25. Let $k$ be any nonzero integer, $B \subseteq X^{\omega}$ be non-self dual. If $B \in$ $\mathbf{B C}(\mathbf{k})$, then either $B$ or $B^{\complement}$ is Wadge equivalent to some

$$
\Omega(\alpha)=\left(\emptyset \bullet \omega_{1}^{n_{j}}\right) \bullet \delta_{j}+\left(\emptyset \bullet \omega_{1}^{n_{j-1}}\right) \bullet \delta_{j-1}+\ldots+\left(\emptyset \bullet \omega_{1}^{n_{0}}\right) \bullet \delta_{0} .
$$

where $j \in \mathbb{N}, n_{j}>n_{j-1}>\ldots>n_{0}$ and $\omega^{k+1}>\delta_{j}, \delta_{j-1}, \ldots, \delta_{0}>0$.

Proof. This is an almost immediate consequence of Lemmas 22 and 24.

This settles the case of the non-self dual $\omega$-languages in $\mathbf{B C}(\mathbf{k})$. For the selfdual ones, it is enough to notice the easy following: 
(a) Given any $A \subseteq X^{\omega}$, if $A \in \mathbf{B C}(\mathbf{k})$ is self dual, then there exists two non-self dual sets $B, C \subseteq X^{\omega}$ such that both $B$ and $C$ belong to $\mathbf{B C}(\mathbf{k}), B \equiv{ }_{w} C^{\complement}$, and $A \equiv_{w} X_{0} B \cup X_{1} C$, where $\left\{X_{0}, X_{1}\right\}$ is any partition of $X$ in two nonempty sets.

(b) If $A \subseteq X^{\omega}$ and $B \subseteq X^{\omega}$ are non-self dual, satisfy $A \equiv{ }_{w} B^{\complement}$, and both belong to $\mathbf{B C}(\mathbf{k})$, then, given any partition of $X$ in two non-empty sets $\left\{X_{0}, X_{1}\right\}, X_{0} A \cup X_{1} B$ is self-dual, and also belongs to $\mathbf{B C}(\mathbf{k})$.

As a consequence, we obtain the following general result if we come back to the original definition of the Wadge degree of a set (denoted $d^{\circ}$ ) - from which we slightly departed from to define $d_{W}$ - namely:

Definition 13. For $A \subseteq X^{\omega}$, we set

$$
d^{\circ}(A)=\sup \left\{d^{\circ}(B)+1 \mid B<_{W} A\right\} .
$$

(Notice that this definition implies $d^{\circ}(\emptyset)=d^{\circ}\left(\emptyset^{\complement}\right)=0$.)

Theorem 26. For any $A \subseteq X^{\omega}$, there exists an $\omega$-language $B \subseteq X^{\omega}$ recognized by some deterministic Petri net, such that $A \equiv_{w} B$ if and only if $d^{\circ} A$ is of the form

$$
\alpha=\omega_{1}^{n} \cdot \delta_{n}+\ldots+\omega_{1}^{0} \cdot \delta_{0} .
$$

for some $n \in \mathbb{N}$, and $\omega^{\omega}>\delta_{n}, \ldots, \delta_{0} \geq 0$.

From where we immediately obtain the following:

Corollary 27. The height of the Wadge hierarchy of $\omega$-languages recognized by deterministic Petri nets is $\left(\omega^{\omega}\right)^{\omega}=\omega^{\omega^{2}}$.

\section{Conclusions}

We provided a description of the extension of the Wagner hierarchy from automata to deterministic Petri Nets with Muller acceptance conditions. Of course the results would be rigorously the same if we replace Muller acceptance conditions with parity acceptance conditions. But with Büchi acceptance conditions instead, it becomes even simpler since the $\omega$-languages are no more boolean combinations of $\boldsymbol{\Sigma}_{2}^{0}$-sets, but $\boldsymbol{\Pi}_{2}^{0}$-sets. So, the whole hierarchy comes down to the following:

Corollary 28. For any $A \subseteq X^{\omega}$, there exists an $\omega$-language $B \subseteq X^{\omega}$ recognized by some deterministic Petri net with Büchi acceptance conditions, such that $A \equiv_{w}$ $B$ if and only if either 
$\circ d^{\circ} A=\omega_{1}$, and $A$ is $\Pi_{2}^{0}$-complete, or

$\circ d^{\circ} A<\omega^{\omega}$.

Deciding the degree of a given $\omega$-language in $\mathbf{B C}(\mathbf{k})$, for $k \geq 2$, recognized by some deterministic Petri net - either with Büchi or Muller acceptance conditions, remains an open question. Notice that for $k=1$ this decision problem has been shown to be decidable in [13].

Another rather interesting open direction of research is to go from deterministic to non-deterministic Petri nets. It is clear that this step forward brings new Wadge classes - for instance there exist $\omega$-languages recognized by non-deterministic Petri nets with Büchi acceptance conditions that are $\Sigma_{3}^{0}$-complete, hence not $\Delta_{3}^{0}$, [21] but the description of this whole hierarchy still requires more investigations.

\section{References}

[1] H. Carstensen. Infinite behaviour of deterministic Petri nets. In Proceedings of Mathematical Foundations of Computer Science 1988, volume 324 of Lecture Notes in Computer Science, pages 210-219. Springer, 1988.

[2] O. Carton and D. Perrin. Chains and superchains for $\omega$-rational sets, automata and semigroups. International Journal of Algebra and Computation, 7(7):673-695, 1997.

[3] O. Carton and D. Perrin. The Wagner hierarchy of $\omega$-rational sets. International Journal of Algebra and Computation, 9(5):597-620, 1999.

[4] R. Cohen and A. Gold. $\omega$-computations on Turing machines. Theoretical Computer Science, 6:1-23, 1978.

[5] J. Duparc. La forme Normale des Boréliens de rang finis. PhD thesis, Université Paris VII, 1995.

[6] J. Duparc. Wadge hierarchy and Veblen hierarchy: Part 1: Borel sets of finite rank. Journal of Symbolic Logic, 66(1):56-86, 2001.

[7] J. Duparc. A hierarchy of deterministic context free $\omega$-languages. Theoretical Computer Science, 290(3):1253-1300, 2003.

[8] J. Duparc, O. Finkel, and J.-P. Ressayre. Computer science and the fine structure of Borel sets. Theoretical Computer Science, 257(1-2):85-105, 2001.

[9] J. Duparc, O. Finkel, and J.-P. Ressayre. The Wadge hierarchy of Petri nets $\omega$-languages. In Proceedings of the International Symposium on Logical Foundations of Computer Science, LFCS 2013, volume 7734 of Lecture Notes in Computer Science, pages 179-193. Springer, 2013.

[10] J. Engelfriet and H. J. Hoogeboom. X-automata on $\omega$-words. Theoretical Computer Science, 110(1):1-51, 1993.

[11] J. Esparza. Decidability and complexity of Petri net problems, an introduction. Lectures on Petri Nets I: Basic Models, pages 374-428, 1998.

[12] H. Fernau and R. Stiebe. Blind counter automata on $\omega$-words. Fund. Inform., 83(1-2):51-64, 2008. 
[13] O. Finkel. An effective extension of the Wagner hierarchy to blind counter automata. In Proceedings of Computer Science Logic, 15th International Workshop, CSL 2001, volume 2142 of Lecture Notes in Computer Science, pages 369-383. Springer, 2001.

[14] O. Finkel. Topological properties of omega context free languages. Theoretical Computer Science, 262(1-2):669-697, 2001.

[15] O. Finkel. Wadge hierarchy of omega context free languages. Theoretical Computer Science, 269(1-2):283-315, 2001.

[16] O. Finkel. Borel hierarchy and omega context free languages. Theoretical Computer Science, 290(3):1385-1405, 2003.

[17] O. Finkel. Borel ranks and Wadge degrees of context free omega languages. Mathematical Structures in Computer Science, 16(5):813-840, 2006.

[18] O. Finkel. Wadge degrees of infinitary rational relations. Special Issue on Intensional Programming and Semantics in honour of Bill Wadge on the occasion of his 60th cycle, Mathematics in Computer Science, 2(1):85-102, 2008.

[19] O. Finkel. The complexity of infinite computations in models of set theory. Logical Methods in Computer Science, 5(4:4):1-19, 2009.

[20] O. Finkel. Highly undecidable problems for infinite computations. Theoretical Informatics and Applications, 43(2):339-364, 2009.

[21] O. Finkel. On the topological complexity of $\omega$-languages of non-deterministic Petri nets. 2012. Preprint.

[22] O. Finkel. Topological complexity of context free $\omega$-languages: A survey. In Language, Culture, Computation: Studies in Honor of Yaacov Choueka, Lecture Notes in Computer Science. Springer, 2013. To appear, available from http://fr.arxiv.org/abs/0806.1413.

[23] S. Greibach. Remarks on blind and partially blind one way multicounter machines. Theoretical Computer Science, 7:311-324, 1978.

[24] J. E. Hopcroft, R. Motwani, and J. D. Ullman. Introduction to automata theory, languages, and computation. Addison-Wesley Publishing Co., Reading, Mass., 2001. Addison-Wesley Series in Computer Science.

[25] A. S. Kechris. Classical descriptive set theory. Springer-Verlag, New York, 1995.

[26] K. Kuratowski. Topology. Academic Press, New York, 1966.

[27] L. Landweber. Decision problems for $\omega$-automata. Mathematical Systems Theory, 3(4):376-384, 1969.

[28] H. Lescow and W. Thomas. Logical specifications of infinite computations. In J. W. de Bakker, W. P. de Roever, and G. Rozenberg, editors, A Decade of Concurrency, volume 803 of Lecture Notes in Computer Science, pages 583-621. Springer, 1994.

[29] D. A. Martin. Borel determinacy. The Annals of Mathematics, 102(2):363-371, 1975.

[30] Y. N. Moschovakis. Descriptive set theory, volume 155. American Mathematical Society, 2009.

[31] R. M. Naughton. Testing and generating infinite sequences by a finite automaton. Information and Control, 9:521-530, 1966.

[32] D. Perrin and J.-E. Pin. Infinite words, automata, semigroups, logic and games, volume 141 of Pure and Applied Mathematics. Elsevier, 2004.

[33] G. Rozenberg. Lectures on concurrency and Petri nets: advances in Petri nets, volume 3098. Springer Verlag, 2004. 
[34] V. Selivanov. Fine hierarchy of regular $\omega$-languages. In Proceedings of the International Joint Conference on the Theory and Practice of Software Development TAPSOFT-95, in Aarhus, Denmark, volume 915 of Lecture Notes in Computer Science, pages 277-287. Springer, 1995.

[35] V. Selivanov. Fine hierarchy of regular $\omega$-languages,. Theoretical Computer Science, 191:37-59, 1998.

[36] V. Selivanov. Wadge degrees of $\omega$-languages of deterministic Turing machines. In Proceedings of the International Conference STACS 2003, 20th Annual Symposium on Theoretical Aspects of Computer Science, Berlin, Germany, volume 2607 of Lecture Notes in Computer Science, pages 97-108. Springer, 2003.

[37] V. Selivanov. Wadge degrees of $\omega$-languages of deterministic Turing machines. RAIROTheoretical Informatics and Applications, 37(1):67-83, 2003.

[38] V. Selivanov. Fine hierarchies and m-reducibilities in theoretical computer science. Theoretical Computer Science, 405(1-2):116-163, 2008.

[39] V. Selivanov. Fine hierarchy of regular aperiodic omega-languages. International Journal of Foundations of Computer Science, 19(3):649-675, 2008.

[40] V. Selivanov. Wadge reducibility and infinite computations. Special Issue on Intensional Programming and Semantics in honour of Bill Wadge on the occasion of his 60th cycle, Mathematics in Computer Science, 2(1):5-36, 2008.

[41] W. Sierpinski. Cardinal and ordinal numbers. PWN (Warszawa), 1965.

[42] P. Simonnet. Automates et théorie descriptive. PhD thesis, Université Paris VII, 1992.

[43] L. Staiger. Hierarchies of recursive $\omega$-languages. Elektronische Informationsverarbeitung und Kybernetik, 22(5-6):219-241, 1986.

[44] L. Staiger. Research in the theory of $\omega$-languages. Journal of Information Processing and Cybernetics, 23(8-9):415-439, 1987. Mathematical aspects of informatics (Mägdesprung, 1986).

[45] L. Staiger. $\omega$-languages. In Handbook of formal languages, Vol. 3, pages 339-387. Springer, Berlin, 1997.

[46] L. Staiger and K. Wagner. Rekursive Folgenmengen. I. Z. Math. Logik Grundlag. Math., 24(6):523-538, 1978.

[47] W. Thomas. Automata on infinite objects. In J. van Leeuwen, editor, Handbook of Theoretical Computer Science, volume B, Formal models and semantics, pages 135-191. Elsevier, 1990.

[48] R. Valk. Infinite behaviour of Petri nets. Theoretical computer science, 25(3):311-341, 1983.

[49] W. Wadge. Reducibility and determinateness in the Baire space. $\mathrm{PhD}$ thesis, University of California, Berkeley, 1983.

[50] K. Wagner. On $\omega$-regular sets. Information and Control, 43(2):123-177, 1979.

[51] T. Wilke and H. Yoo. Computing the Wadge degree, the Lifschitz degree, and the Rabin index of a regular language of infinite words in polynomial time. In P. Mosses, M. Nielsen, and M. Schwartzbach, editors, TAPSOFT 95, volume 915 of Lect. Notes in Comp. Sci., pages 288302. Springer Verlag, Berlin, Heidelberg, New York, 1995. 